\title{
Measurement of Internal Heat Transfer Distribution of Highly-Loaded Gas Turbine Blade by Combined Experi- mental/Numerical Method
}

\author{
Asif $\mathrm{Ali}^{1, *}$, Lorenzo Cocchi ${ }^{1}$, Alessio Picchi ${ }^{1}$, and Bruno Facchini ${ }^{1}$ \\ ${ }^{1}$ Department of Industrial Engineering, University of Florence, 50139, via S.Marta 3, Florence, Italy
}

\begin{abstract}
To ensure a passable life span of gas turbine hot gas path components the measurement of metal surface temperature is paramount. Experimental analyses on internally cooled devices are often performed on simplified or scaled up geometries, which reduces the applicability of the results to the actual real hardware. A more reliable estimation of cooling performance could be obtained if the real engine component is directly studied. To achieve this goal, an experimental campaign is performed to investigate the internal heat transfer distribution of an industrial blade, cooled by means of an internal U-shaped channel. During the experiment the blade is heated to a known temperature, then a coolant is introduced through the internal channel to induce a thermal transient, during which the external surface temperature is measured with the help of an infrared camera. Then a transient thermal finite element simulation is performed with the same boundary and inlet conditions of the experiment. Based on the output of the simulation, the internal heat transfer distribution is updated until convergence between simulation output external temperature and the experimental temperature is achieved. In order to start the iterative procedure, a first attempt estimation of the internal heat transfer distribution is obtained with a lumped thermal capacitance model approach. Different experiments were performed with different mass flow rates and the results are compared with available literature data. The obtained results allow to observe detailed heat transfer phenomena, strongly bound to the relevant features of the actual real cooling system.
\end{abstract}

\section{Introduction}

The will of increasing gas turbine performance levels lead to growing values of turbine inlet temperature, which nowadays can significantly exceed the material temperature limits. In order to improve the life span of the gas turbine components various internal and external cooling schemes are used. To make these cooling schemes more efficient different cooling configurations are employed i.e. ribs, pins, dimples and impingement jets among others. More detailed review on the cooling enhancement techniques is given in book by Webb [1] and the developments in the gas turbine cooling are enlisted in the book by Han $[2,3]$. Quantitative analysis of any gas turbine component cooling scheme is performed by predicting its heat transfer coefficient. Different methods are available for this purpose including

*e-mail: asif.ali@unifi.it 
Thermochromic liquid crystal (TLC), Infared thermogarphy (IR), flow check and sublimation technique. Earlier work performed by Hylton [4] uses TLC to measure the external surface temperature of the gas turbine blade. But in the recent years the usage of IR for this purpose is increased day by day because of its lower sensitivity to illumination and viewing angle [5]. Different researchers used IR thermography for measuring the heat transfer coefficient of a cooled geometry. Bantel and Mack [6] presented a methodology in which they forced the hot gas to pass through a channel and then observed the transient response of the channel with the help of IR camera. IR thermography was also used for quantitative evaluation of defects in any metal surface and gas turbine blade [7, 8]. Cocchi et al. [9] and Goodro et al. [10] uses IR thermography method for analysis of the impingement jets. A detailed study regarding the use of IR for heat transfer measurement is done by Carlomagno and Cardone [11].

Nirmalan et al. [12] was the first one who made an effort to find a complete internal heat transfer distribution of a metallic airfoil. In this work, an iterative inverse conduction scheme was implemented by running finite element model (FEM) of the geometry, with the goal to numerically replicate the measured thermal transient. The heat transfer distribution will be the one, which provides the same external temperature distribution at a given time step in both the experiment and the FEM analysis. Although a constant coolant temperature was considered, the authors suggested to use a proper fluid model to evaluate local time-varying flow temperature for more complex geometries. In subsequent work [13] the author also extended this technique to quantify the flow features of film cooling holes by comparing the results with a known standard one.

The above short review highlights the use of IR for the measurement of heat transfer of any cooling scheme but most of the investigations were done on the scaled model of the geometry, operated close to the ambient temperature. In this way, however, many significant features of the real engine component were either lost or very difficult to replicate, including surface roughness, deviations from ideal geometry, whole system interactions and others. All of these mentioned features could be maintained if a direct investigation on the real hardware would be performed. There is a growing interest in the researcher community regarding the possibility of performing heat transfer measurement at real scale, high conductivity sample. The goal of the present work, which fits within the research trend, is to develop and implement a measurement procedure for calculating the heat transfer coefficient of internal cooled real geometry.

\section{Methodology}

The proposed methodology is based upon the Nirmalan technique [12] used for the measurement of heat transfer of a component housing an internal cooling scheme. The basic principle of the methodology is to force the internal heat transfer to induce a thermal transient in the component. Then a numerical simulation is set up and the heat transfer distribution is found as the one which makes the simulation behave as experiment.

The geometry for which the heat transfer distribution calculation is required must be first fixed on a test stand and heated to known thermal state $(t=0)$. Then coolant is introduced into the internal cooling system to induce a thermal transient, while the external surface temperature $\left(T_{s}(x, y, z)\right)$ of the geometry is recorded with the help of Infrared (IR) camera.

The output of the above experiment, i.e. the outer surface temperature evolution of the geometry, is used as an input for the numerical procedure with the goal to replicate the same thermal phenomena as the experiment. The schematic of this numerical procedure is shown in Figure 1.

A FEM of the same geometry is set up using the same material properties with the same known boundary conditions of the experiment like initial temperature and coolant flow rate 


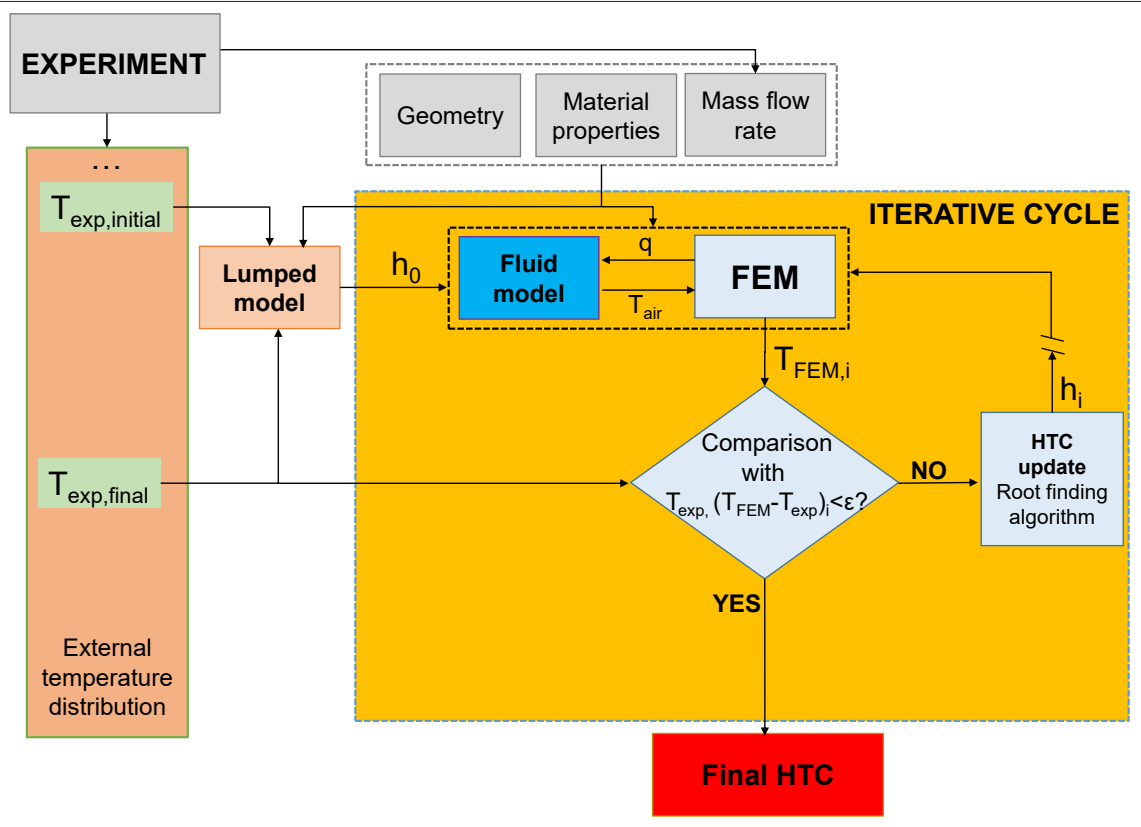

Figure 1. Scheme of the proposed methodology

as well as its temperature and executed for the same thermal transient duration as the experimental one. The FEM is implemented initially with internal heat transfer coefficient (HTC) obtained using lumped thermal capacitance model (LTCM) [14, 15] shown in equation 1.

$$
h=-\frac{\rho c l}{\Delta t} \log \left(\frac{T_{s}-T_{\text {air }}}{T_{\text {in }}-T_{\text {air }}}\right)
$$

where $h$ is the heat transfer coefficient, $\rho$ is the density, $c$ is the specific heat, $l$ is the local wall thickness, $\Delta t$ is the total transient duration, $T_{s}$ is the external surface temperature of the geometry recorded at a time instant, $T_{\text {air }}$ is the coolant temperature and $T_{\text {in }}$ is the initial surface temperature of the geometry at the start of transient test.

The coolant temperature inside the internal cooling channel of the geometry changes continuously during the thermal transient. In order to allow a continues update of the coolant temperature, the total transient test duration is divided into substeps i.e. a total transient test duration of $32 \mathrm{~s}$ is divided into $4 \mathrm{~s}$ substeps. For each substep the FEM is executed with the same HTC value but with other updated boundary conditions like initial temperature and coolant temperature. The initial temperature for the first substep is obtained from the IR data, after that the final FEM output temperature of each previous substep acts as input for the next substep. The inlet and outlet temperatures of the geometry cooling channel are continuously measured with thermocouples during the transient test. One way is to update the cooling channel flow temperature is by linear interpolating the temperature values from the inlet to the outlet value at each substep but thus may not provide an accurate temperature distribution pattern of the flow. Another way is the use of a fluid model, which will find the overall temperature distribution pattern of the coolant inside the geometry. Different fluid models from zero-dimensional to a complex CFD, can used for this purpose. However satisfactory results can also be obtained by using a simple model based upon energy balance. In particular, given that the mass flow rate at each section of the geometry is known, the internal heat surface can 
be divided into sectors, each with single inlet and single outlet for the flow. If the heat flux $q$ and inlet flow temperature $T_{\text {in }}$ are known, energy balance allows to retrieve the outlet flow temperature of the sector $\mathrm{S}$ :

$$
T_{\text {out }}=T_{\text {in }}+\frac{\int_{s} q d S}{m c_{p}}
$$

In the present case, the local heat flux $q$ is provided by the FEM simulation. By starting from the section closer to the inlet and from the measured inlet temperature value, this method allows to retrieve the whole flow temperature distribution along the cooling geometry. If the outlet temperature of the model is also measured, this value can be compared with the one predicted from the model, thus allowing to verify the reliability of the model itself or otherwise to apply a suitable correction.

After running the FEM for the last substep of thermal transient, the output external surface nodal temperature obtained from it will be compared with the experimental external surface temperature. Now if the initial LTCM HTC represents the reality then both outputs will be the equal (whithin a given tolerance) otherwise the HTC value has to be modified until the convergence between them is achieved. A root finding algorithm can be used for iteration purpose, e.g. using a secant method shown in the equation 3.

$$
h_{i+1}=h_{i}-\frac{\text { Tempdiff }}{\text { Tempdiff } f_{i}-\text { Tempdiff }_{i-1}}\left(h_{i}-h_{i-1}\right)
$$

Where $h_{i+1}$ is the updated heat transfer coefficient value, $h_{i}$ and $h_{i-1}$ are the HTC values from the two previous iterations while Tempdiff $f_{i}$ and Tempdif $f_{i-1}$ are the temperature differences between FEM and experimental values obtained at these iterations. As observed, the secant method will need two starting HTC values so the first one will be the LTCM one while the other can be chosen as multiple of LTCM i.e $0.9 \times H T C_{L T C M}$ (if $T_{\text {exp }}$ is less than $T_{F E M}$ ) or $1.1 \times H T C_{L T C M}$. The whole iteration process will be continued until the convergence between the FEM and experimental external surface temperature is obtained i.e. the local temperature difference between them is lower than a given fixed threshold.

\section{Experimental Setup}

\subsection{Test Rig}

The proposed methodology is implemented on a geometry with the help of a suitable test rig installed in the Heat Transfer and Combustion Laboratory of the Department of Industrial Engineering of the University of Florence. Figure 2 shows the rig scheme while pictures of the whole assembly are visible in Figure 3.

As discussed that the first step of our methodology is to induce a controlled thermal transient, so for this purpose an oven is employed. Ten ceramic heaters are housed inside the oven, each one capable of providing an output power of $325 \mathrm{~W}$, fed by a $4.8 \mathrm{kVA}$ variac. For measuring the surface temperature of the geometry during the transient, a pliers-like design is used for the oven: the heaters are installed on two semi-cylindrical shutters, which can be manually closed to embrace the test article during the heating phase or opened to expose it to the camera view during the test. Also the oven is provided with the rail system which helps it in moving away from the geometry after heating phase thus exposing geometry to the view of an IR camera.

The coolant air is provided by the air supply system which has the capability of providing up to $40 \mathrm{~g} / \mathrm{s}$ of dry air. For having pure and pressurized air flow, the air supply system is provided with filtering section and pressure regulator. The flow is regulated with the help of 


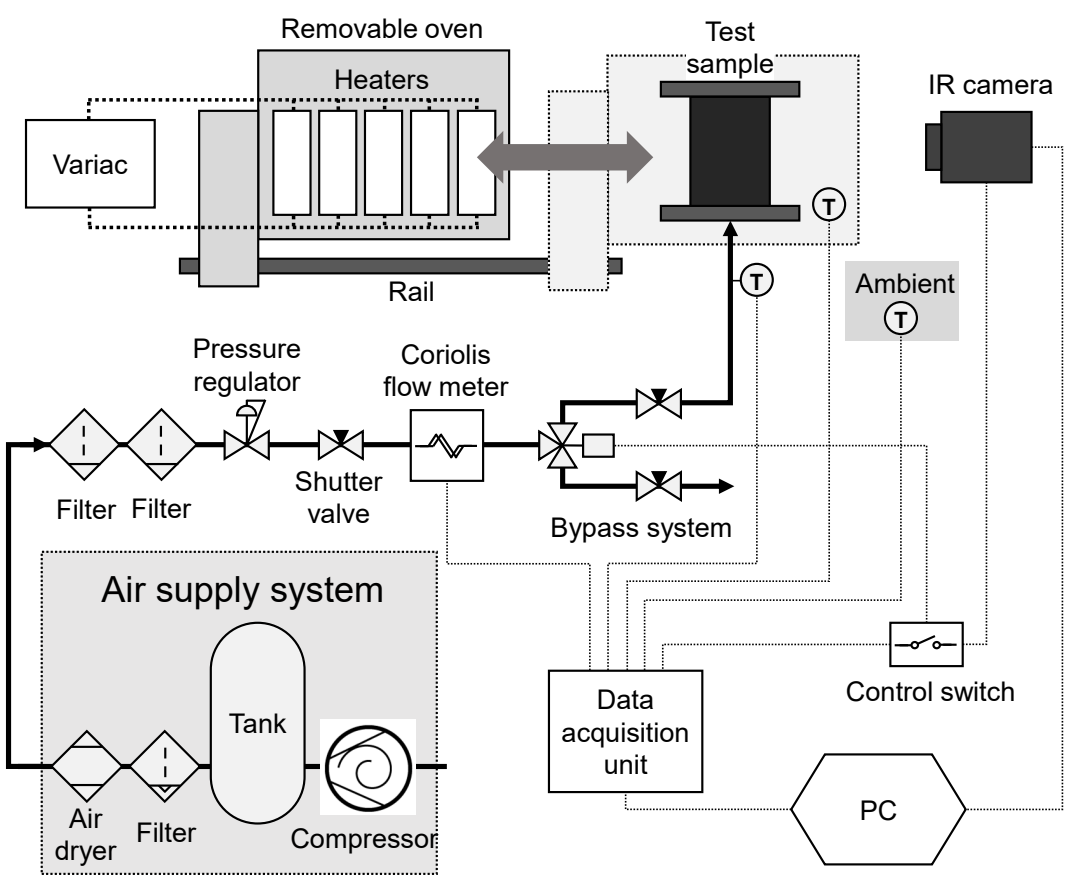

Figure 2. Test rig scheme

a shutter valve which is then followed by Coriolis flow meter for measuring the flow rate. A Bronkhorst CORI - FLOW ${ }^{T M}$ M55 Coriolis flow meter with a $12 \mathrm{~g} / \mathrm{s}$ range and accuracy of $0.5 \%$ is used. A bypass system was also introduced for air supply and measurement along the flow path to avoid sudden mass flow variation at the beginning of test for the upstream supply and measurement equipment. In the bypass system the feeding line is split into two branches by means of an actuated 3-way valve, one branch is connected to the test sample while the other one, bypasses the air to the environment. Both branches are provided with shutter valves which are set in such a way that the total head loss remains the same. In this way, the desired flow conditions are set before the test by flowing air through bypass line. By activating the 3-way valve the flow is directed toward the test article, but this doesn't cause any apparent changes for the upstream system.

As our test article is provided with a support and a perforated part which helps in fixing the article in inside the test rig. The external surfaces of the test article are painted with a high temperature resistant black paint to increase its emissivity and to improve the measurement of the surface temperature. The emissivity of the used paint is calibrated with the help of dedicated apparatus consisting of a flat aluminium bar painted on one side and heated by a film heater on the other side. A thermocouple is also inserted inside the bar whose readings were compared with the IR temperature readings of the bar surface for retrieving the emissivity values. It is also observed that the emissivity value remains constant up to $60^{\circ}$ viewing angles. For having a uniform emissivity value the region with viewing angle greater than $60^{\circ}$ are excluded from the postprocessing analysis and also the test article is positioned to avoid such regions.

Our methodology needed a full temperature distribution of the test article surface during the transient phase, achieved with the help of an IR camera and two mirrors. The IR is focused 
directly on the leading edge (LE) while pressure side (PS) and suction side (SS) views are observed through the mirrors. The mirrors are made of PMMA smooth plates where a thin

(a)

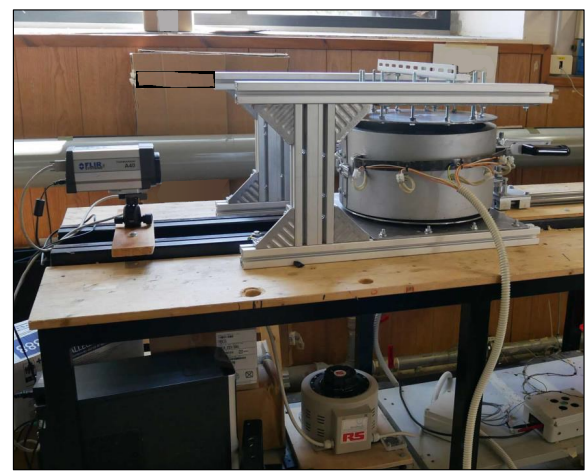

(b)

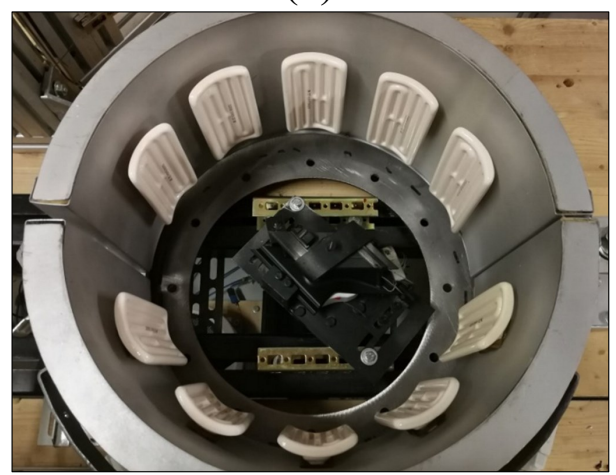

Figure 3. (a)Test rig overall assemble (b) Test article install inside the oven

aluminium layer is deposited for reflecting the IR radiations. Both mirrors are provided with movable stands which move them to move quickly to the desired position before the test starts. A FLIR ThermoVision ${ }^{T M}$ A40 IR camera provided with Focal Plane Array (FPA) uncooled microbolometer is used for measuring the article surface temperature. The IR camera is set to record a $240 \times 320$ points stream at $25 \mathrm{~Hz}$. Two T type thermocouples are installed at inlet and outlet of the geometry cooling channel to measure the temperature of the coolant air during the test. Other thermocouples are also installed for measuring the ambient temperature and some specific location temperature inside the oven during the heating phase for monitoring purpose.

\subsection{Test article}

A prismatic blade model 3D printed in Inconel is used to perform measurements. The blade profile has been built by extruding the midspan profile of a first stage rotor blade by considering a reduction scale factor of 1.5 .

The blade has an internal cooling scheme which consist of U-bend channel as shown in the figure 4(a). It is provided with perforated flange which helps in fixing the article in the test rig. As shown in figure 4(a) the cooling flow is fed through the LE channel. The U-bend region of the channel is shown transparent to provide a clear view of the coolant path and the deviator in this region. The goal of this measurement campaign is to calculate the internal heat transfer coefficient distribution of the U-bend channel by using infrared thermography.

\subsection{Experimental procedure}

The first step is the heating of the test article inside the oven to a known constant temperature, by considering $220{ }^{\circ} \mathrm{C}$ as the target temperature. During the heating phase the temperature inside the oven and temperature of test article are constantly monitored with help of the installed thermocouples. The temperature of the test article is considered as steady once the temperature variation measured by each thermocouple is lower than $0.001 \mathrm{~K} / \mathrm{s}$. Once the temperature is steady, the air flow is initiated through the bypass line and the desired mass 
(a)

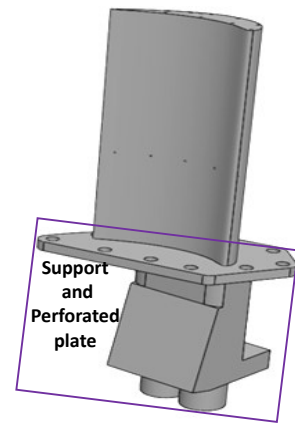

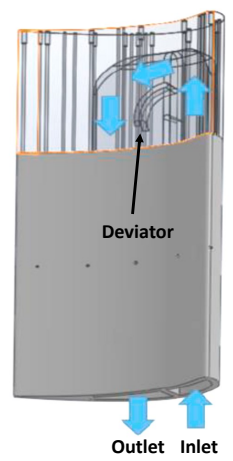

(b)

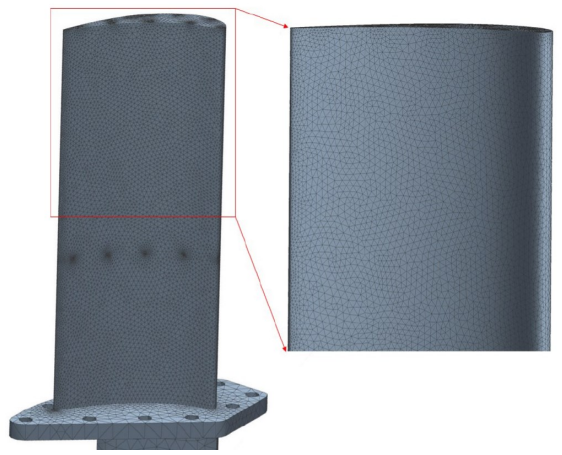

Figure 4. (a)Test article with internal U-bend smooth channel (b) Thermal grid details of article

flow rate is set. Then the oven is quickly removed and the mirrors are moved to the desired position. A designated switch triggers the start of the test by swapping the 3-way value (which allows the coolant to flow inside test article) and activates the IR camera and the acquisition unit for recording the data. A test duration of $120 \mathrm{~s}$ is considered which will be enough to capture the most significant region of the thermal transient for all investigating conditions.

\subsection{Data postprocessing}

To implement the $3 \mathrm{D}$ postprocessing, the $2 \mathrm{D}$ temperature data recorded by the IR camera must be associated to the corresponding location of the test article outer surfaces. A customized 3D mapping procedure was developed for this purpose which associates a series of marker points on the test sample to the known position of the solid model of the geometry and thus provides the $(\mathrm{x}, \mathrm{y}, \mathrm{z})$ position of every relevant point of the IR camera output.

The postprocessing procedure which is already discussed in the previous sections is applied with Matlab. A customized Matlab macro was developed to implement all of the step of the postprocessing procedure except the FEM part which is carried out with ANSYS Mechanical v18 via automatic batch execution. FEM model used the test article CAD geometry and consists of about $2.179 \times 10^{5}$ solid87 elements (ten nodes tetrahedrons elements). The grid of the airfoil is properly refined to characterize each detail of the internal cooling scheme without significantly affecting the computational time (figure 4(b)). In the solver, temperature dependent material properties like density, specific heat and thermal conductivity were implemented. Heat transfer coefficient value was set on the internal surface of the channel, whose value was updated along the iterations as results of the procedure. External convection and radiation were imposed on the outer surface of test article to model the heat losses towards the environment. In particular, the external convective heat transfer coefficient value is retrieved from literature correlations [14] and its value was set constant and equal to the average evaluated along with the test duration. Because the value never exceed more than $6 \%$ of the local internal heat transfer even in the case of smallest Re value, this approximation is considered as satisfactory for the present case.

Concerning the convergence criterion, root mean square value of the temperature difference between the measured and calculated temperature must fall below $0.6 \mathrm{~K}$, which is achieved after 4 to 5 iterations. Around 11 to 12 hours will be required for the whole postprocessing 
procedure when it is run on a desktop personal computer (Intel Core ${ }^{T M}$ i7-6700 CPU, $32 \mathrm{~Gb}$ RAM).

\section{Results}

The final heat transfer distribution is shown in the figure 5, which is obtained by implementing the proposed methodology on the test article considering the largest investigated Re value ( $R e=\frac{m D}{A \mu}$ : where $m$ is the mass flow rate, $D$ is the channel hydraulic diameter considered as reference length, $A$ is the effective area and $\mu$ is the viscosity evaluated at the average film temperature during the test). The transfer coefficient $h$ is recast in a dimensionless form as Nusselt number using the following equation:

$$
N u=\frac{h D}{k}
$$

where $k$ is the air thermal conductivity which is evaluated at the average film temperature during the test. In Figure 5, the $N u$ is scaled with respect to the average value of the LE channel $N u_{r e f}$. In the figure only the section of interest of the cooling system (i.e. the one
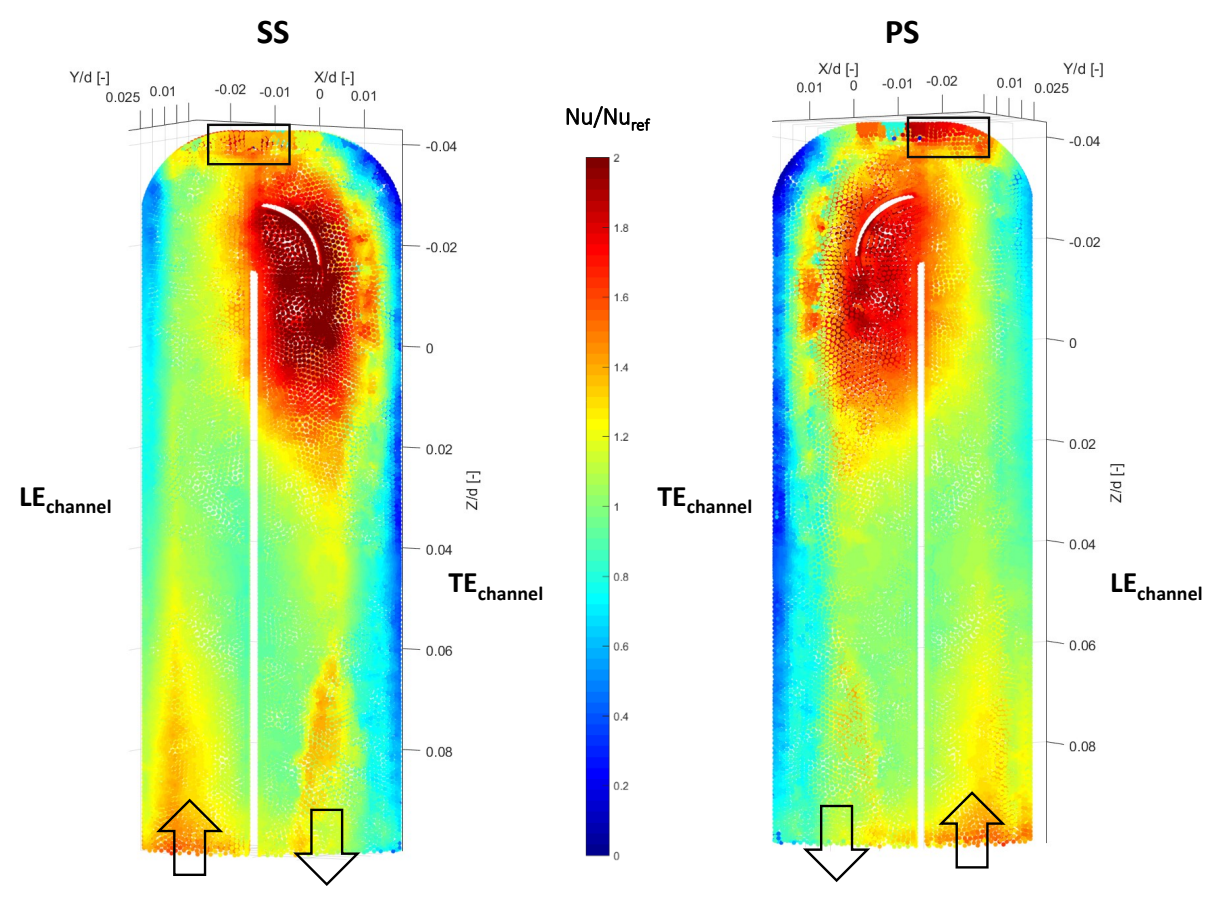

Figure 5. $N u$ distribution for $R e=36870$ on both suction and pressure side

actually lying inside the airfoil) is reported.

The heat transfer pattern and magnitude related to the specific features of the internal cooling U-bend channel can be easily noted from the obtained result with a satisfactory resolution. LE channel heat transfer values are almost uniform along the channel, until reaching the Ubend region where the values become larger. In particular, a strongly localized heat transfer 
increase can be identified at the top of the cooling geometry, which can be attributed to the flow momentum in the direction of the LE duct driving the flow to impinge in such region [16]. This higher heat transfer value region is highlight by a black rectangular in the Figure 5, a spot is observed where the flow is impinged. Further along the channel the presence of the deviator enhances the velocity of the flow which increases the heat transfer in its surrounding region. This effect extents even to the top region of the tailing edge (TE) channel. Then, as the flow turns back and moves along the TE channel, the values become more uniform and closer to the values of the LE region. Lower heat transfer values are observed in the TE channel in the region closer the TE of the blade, which can be attributed to wedge shape of the channel section. The heat transfer values of the whole U-bend cooling channel are almost having same distribution pattern considering both SS and PS of the blade.

\subsection{Effect of Reynolds number}

Different test cases were considered, each with a different coolant mass flow rate, which correspond to different $R e$ value. A quicker cooling phenomenon was expected for larger internal heat transfer values. As consequence, different test durations were considered for each investigated mass flow rate. For example $32 \mathrm{~s}$ for $R e=36870,48 \mathrm{~s}$ for $R e=28468,64 \mathrm{~s}$ for $R e=19547$ and $80 \mathrm{~s}$ for $R e=10208$. Even so, the whole test duration is divided into the same number of substeps for the postprocessing of the experiment. This results in a different duration of each substep for different Re values, e.g. $4 \mathrm{~s}$ for the case of highest $\mathrm{Re}=36870$ and $8 \mathrm{sec}$ for the lowest $R e=10208$. This was done to keep the wall temperature difference and coolant temperature for a substep to a established value in each test. The obtained final

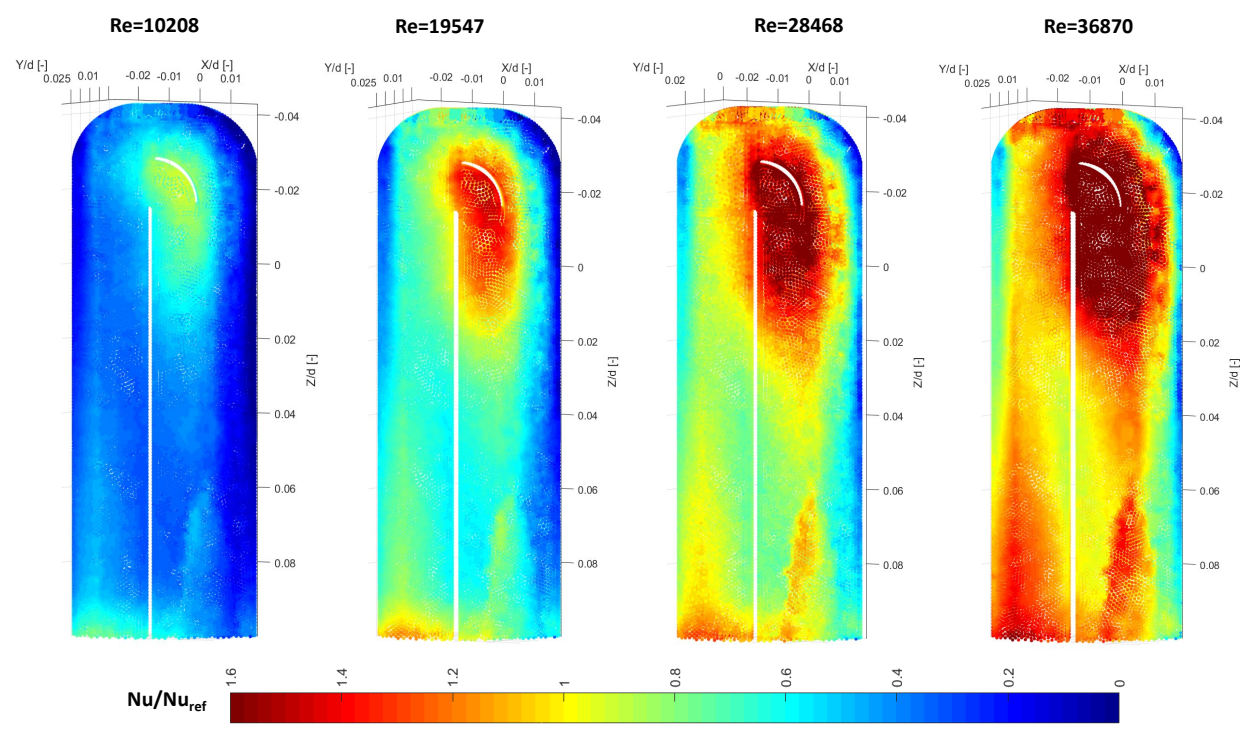

Figure 6. Effect of Re on $\mathrm{Nu}$ distribution

results for all test are shown in the Figure 6. All $N u$ values are scaled with respect to the same $N u_{\text {ref }}$ value defined in the previous section. With each coolant mass flow rate the heat transfer distribution increases monotonically at every location of the U-bend channel, as expected. No significant change to the overall $\mathrm{Nu}$ distribution pattern can be observed: in 
fact, local difference between the shape of the various patterns can be entirely attributed to the post-processioning noise and test repeatabilty.

\subsection{Comparison with available literature data}

To justify the reliability of the obtained results a comparison is performed with the expected heat transfer results obtained from literature data by considering the section of the internal channel preceding the U-bend as a straight duct. In particular, experimental values are averaged over a streamwise section of the LE duct (higlighted in Figure 7 by black rectangular), sufficiently far both from the inlet and the U-bend to suppose that a fully developed flow is present there. In this way, a comparison between the predicted and measured values can be performed. Amongst the available correlations, the obtained results were compared both with the well-known Dittus-Boelter correlation [17] and with Petukhov second equation [18], which also takes into account the effect of roughness on heat transfer. In fact, the additive manufacturing technique used for building our test article entails a surface roughness of 50 $\mu m$ on the cooling channel.

Figure 7 shows the comparison of the measured value: $N u_{-} M$ (where $N u \_M$ is the average value in the LE selected region) with Dittus-Boelter $\left(N u \_D\right)$ and Petukhov second equation $\left(N u_{\_} P\right)$. It is clear from the figure that the measured values are lower or higher just by $2 \%$ to $3 \%$ percent than the Petukhov second equation values for all Re numbers. In Dittus-Boelter cases the value diverge more as moving towards higher $R e$, because the Dittus-Boelter correlation doesn't consider the friction factor effect on the heat transfer value, which increases moving towards higher $R e$ values. Figure 7 also shows that the measured values are directly proportional to $R e^{0.84}$, closely following the trend $N u \propto R e^{0.8}$ which further proves its reliability.
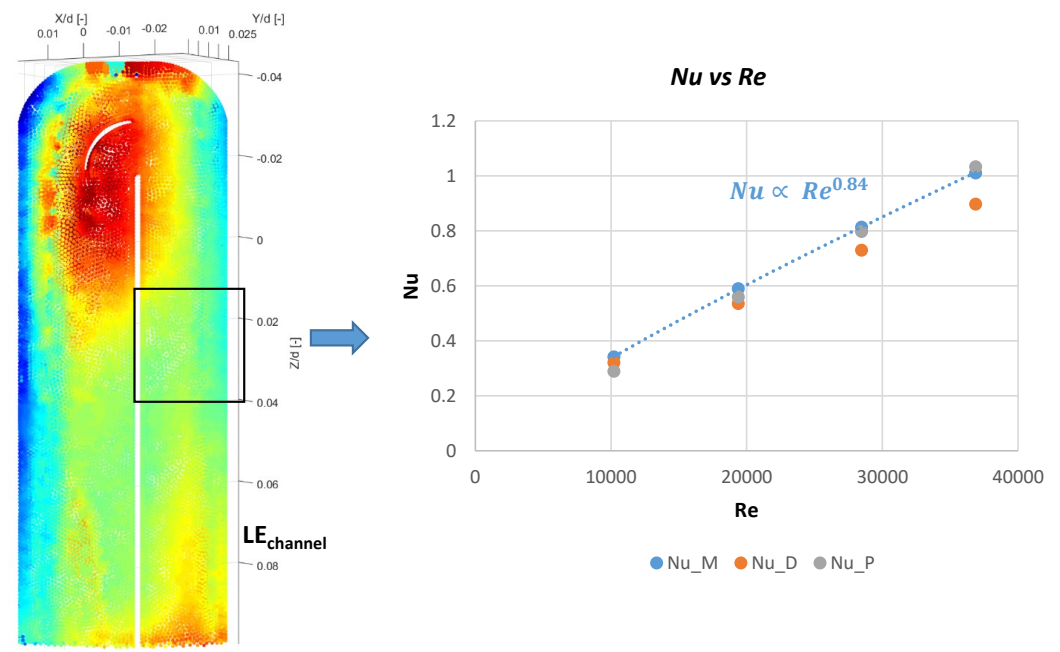

Figure 7. $N u$ vs $R e$ for a selected LE region

\section{Uncertainty analysis}

Because of the complexity of the postprocessing procedure the measurement uncertainty estimation can be only done by considering each input uncertainty and performing the sensitivity 
of the outcome $[19,20]$. The corresponding uncertainty is added to each input parameter and the obtained output is compared with already obtained undistributed one (Figure 5). The obtained results of such analysis are enlisted in the following figure 8(a). The contribution is split between LE and TE channel to check the effect of parameter uncertainty on these regions.

To retrieve an estimation of the global error on $\mathrm{Nu}$ related to each parameter, all contributions are combined using following quadratic error propagation model (equation 5).

$$
\varepsilon_{t o t}=\sqrt{\left(\varepsilon_{1}^{2}\right)+\left(\varepsilon_{2}^{2}\right)+\left(\varepsilon_{3}^{2}\right)+\ldots+\left(\varepsilon_{n}^{2}\right)}
$$

where $\varepsilon_{i}$ represents the relative uncertainty associated with each parameter and $\varepsilon_{t o t}$ is the overall uncertainty. Figure 8(b) shows sample outcome for $R e=36870$ of such analysis. Average uncertainty of $5 \%$ to $7 \%$ is observed both on LE and TE channel. The value increases up to $15 \%$ at the lower passage area region specially on TE of the blade because of the higher effect of the external $h$ on the heat transfer.

(a)

\begin{tabular}{|c|c|c|c|}
\hline \multirow{2}{*}{ Parameter } & \multirow{2}{*}{ Uncertainty } & \multicolumn{2}{|c|}{$\begin{array}{c}\text { Typical contribution to } \\
\text { Nu uncertainty }\end{array}$} \\
\cline { 3 - 4 } & & $\begin{array}{c}\text { LE } \\
\text { Channel }\end{array}$ & $\begin{array}{c}\text { TE } \\
\text { Channel }\end{array}$ \\
\hline$T_{W}$ & $0.6 \mathrm{~K}$ & $4 \%$ & $3.5 \%$ \\
\hline$T_{F}$ & $0.6 \mathrm{~K}$ & $0.75 \%$ & $0.75 \%$ \\
\hline$k$ & $5 \%$ & $4.5 \%$ & $4 \%$ \\
\hline$m$ & $0.5 \%$ & $0.5 \%$ & $1 \%$ \\
\hline$h_{\text {ext }}$ & $15 \%$ & $4 \%$ & $5 \%$ \\
\hline$\rho c$ & $5 \%$ & $4 \%$ & $4 \%$ \\
\hline$t$ & $0.5 \mathrm{~s}$ & $3 \%$ & $2 \%$ \\
\hline
\end{tabular}

(b)

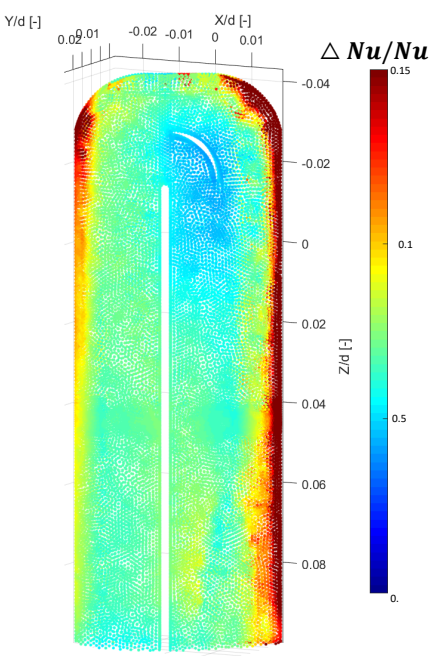

Figure 8. (a) Contribution to uncertainty on $N u$.(b) Local uncertainty for $R e=36870$ case

\section{Conclusion}

In this work, full 3D heat transfer distribution for an internally cooled, additively manufactured metal blade sample are retrieved. To achieve this goal, a transient experimental technique based on the method proposed by Nirmalan [12] is applied. The blade is preheated to a predetermined temperature, then a coolant is introduced into the internal cooling channel which enforces a control thermal transient. During the thermal transient the external surface temperature of the blade is recorded with the help of an IR camera.

The obtained experimental data is then fed to FEM model of the same blade whose goal is to find the internal heat transfer coefficient of the blade internal cooling channel in order to procure external surface temperature as the experimental one. During the thermal transient, 
FEM model is constantly run with updated coolant temperature using fluid model (based upon energy balance) and thermocouples readings fixed at the inlet and outlet of the channel. The acquired results revealed that the methodology is capable of providing significant details about the internal cooling channel heat transfer coefficient distribution and also allows us to assess the effect of different coolant mass flow rate on the heat transfer. The obtained results are validated by comparing the values retrieved on a region of fully developed duct flow with available literature correlations.

Based upon the achieved outcomes, it is concluded that the proposed methodology can be considered a useful technique for investigating any internal cooled real geometry and performing the detail analysis of any applied cooling scheme.

\section{References}

[1] L.R. Webb, NY. Kim, Enhanced heat transfer, (2005)

[2] J.C. Han, S. Dutta and S. Ekkad, Gas turbine heat transfer and cooling technology, (2012)

[3] J.C. Han and M. Huh, Recent studies in turbine blade internal cooling,(2009)

[4] L.D. Hylton, M.S. Mihelc,E.R. Turner,D.A. Nealy and R.E. York, NASA Contractor Report, NASA CR-168015,(1983)

[5] S.V. Ekkad, S. Ou and R.B. Rivir, J. Turbomach, 126, 597-603(2004)

[6] T.E. Bantel and D.C. Mack, US Patent 4,644,162, (1987)

[7] T.E. Bantel, US Patent 5,111,046, (1992)

[8] A. Daniels, Thermosense XVIII: An International Conference on Thermal Sensing and Imaging Diagnostic Applications, 2766, 185-201 (1996).

[9] L. Cocchi, B. Facchini, S. Giuntini, L. Winchler, L. Tarchi, L. Innocenti, L. Andrei and A. Bonini, ASME Turbo Expo 2016: Turbomachinery Technical Conference and Exposition, (2016).

[10] M. Goodro, J. Park, P. Ligrani, M. Fox, Mike and H-K. Moon, Int. J. of Heat and Mass Transf, 50, 367-380 (2007)

[11] G.M. Carlomagno and G. Cardone, Experiments in fluids, 49, 1187-1218(2010)

[12] N.V. Nirmalan, R.S. Bunker and C.R. Hedlund, J. Turbomach., 125, 83-89 (2003)

[13] R.S. Bunker, S.J. Osgood and N.V. Nirmalan, ASME Turbo Expo 2003, collocated with the 2003 International Joint Power Generation Conference, 443-450 (2003)

[14] F. P. Incropera, D. P. DeWitt, T. L. Bergman, and A. S. Lavine, Fundamentals of heat and mass transfer (John Wiley \& Sons, 2007)

[15] M.N. Özışık, Heat transfer: a basic approach, 1, (1985)

[16] L.W. Florschuetz, C.R. Truman and D.E Metzger, J. of Heat Transf.,13, 337-342(1981)

[17] F.W. Dittus and L.M.K. Boelter,International Communications in Heat and Mass Transfer, 12, 3-22(1985)

[18] B.S. Petukhov and others, Advances in heat transfer, 6, 503-564(1970)

[19] P. Heidrich, and J. von Wolfersdorf and M. Schnieder, ASME Turbo Expo 2008: Power for Land, Sea, and Air, 275-286(2008)

[20] M. Kinell, E. Utriainen and P. Jaksch, ASME Turbo Expo 2014: Turbine Technical Conference and Exposition., (2014). 